\title{
A Novel Clinico-Biochemical Score for Screening of Inherited Metabolic Diseases in Children
}

\author{
Oshaque Ali1, Ayesha Hafeez1, Aamir ljaz1, Munir Akmal Lodhi2, Muhammad Asif Nawaz¹ and Zeeshan Ahmed1
}

\begin{abstract}
Objective: To evaluate a novel clinico-biochemical score for screening of inherited metabolic diseases (IMDs) in children in our setup.

Study Design: Descriptive analytical study.

Place and Duration of Study: Department of Chemical Pathology and Endocrinology, Armed Forces Institute of Pathology, Rawalpindi, from August 2016 to August 2017.

Methodology: Clinical data, preliminary biochemical investigations, plasma amino acid (PAA) and organic acid profiles (where indicated) of 354 children, aged $<1$ year to 12 years, referred to the study place for evaluation of suspected inherited metabolic diseases, was collected and evaluated. A clinico-biochemical score card named Rawalpindi Inherited Metabolic Diseases Score (RISc) was devised, on a scale from 1 to 10, incorporating 5 clinical and 5 important biochemical findings, and each variable was assigned a score, based on its relative frequency/risk. Each case was then assigned the RISc score and evaluated for presence or absence of any inherited metabolic disease, based on the score. This score was validated keeping plasma amino acids and organic acid profiles (in selected cases) as reference standard. Results: Patients were divided into three groups, based on RISc score as low RISc (0.5-2.5), medium RISc (3.0-5.5) and high RISc (6-10). A total of 354 cases reported in 2016 and 2017 and $33(9.3 \%)$ were diagnosed to be having IMDs. One (3.0\%) patient from low RISc, four (12.1\%) from medium RISc, and $28(84.8 \%)$ from high RISc group were found to test positive for any one IMD. High RISc group had a statistically significant higher IMD rate than the other two groups $(p<0.001)$. Specificity, sensitivity, positive likelihood ratio, negative likelihood ratio, positive predictive value, negative predictive value and accuracy were $93 \%, 85 \%, 11.8,0.16,55 \%, 98 \%$ and $90 \%$, respectively.

Conclusion: The cost effective RISc, based on clinical data and preliminary biochemical investigations, is highly accurate in diagnosing IMDs in cost restrained setups. It is strongly suggested that the initial screening for suspected IMDs and decision for advanced laboratory testing be carried out, based on the RISc card presented in the study.
\end{abstract}

Key Words: Inherited metabolic disease, Plasma amino acids, Organic acids, Rawalpindi inherited metabolic diseases score.

\section{INTRODUCTION}

Diagnosis of inherited metabolic diseases (IMDs) constitutes a real challenge in a setup like ours, where resource constraints and absence of systematic newborn screening programmes compounded by high consanguinity rate and lack of technical expertise often delay the diagnosis. ${ }^{1}$ IMDs are complex genetic disorders of metabolism, including that of carbohydrate, protein and fats, ${ }^{2}$ and usually have a complex clinical presentation posing challenge for clinician to diagnose. Patients may present with non-specific symptoms of lethargy, poor feeding, convulsions, ${ }^{3}$ failure to thrive, jaundice, ${ }^{4}$ altered acid base status, hyper ammonemia, ${ }^{5}$ and deranged liver function tests (LFTs).

1 Department of Chemical Pathology and Endocrinology, Armed Forces Institute of Pathology, Rawalpindi, Pakistan

2 Department of Paediatrics, Military Hospital, Rawalpindi, Pakistan

Correspondence: Maj. Oshaque Ali, Department of Chemical Pathology and Endocrinology, Armed Forces Institute of

Pathology, Rawalpindi, Pakistan

E-mail: oshaque105443@gmail.com

Received: 19 January 2018; Accepted: July 20, 2018
Various stumbling blocks in diagnosis are the nonspecificity of the signs and symptoms, the need for exclusion of routine childhood illnesses in the first place, ${ }^{6}$ inappropriate sample collection or storage and cost constraints. In a developing country like Pakistan with a high rate of consanguinity, ${ }^{7}$ it is virtually impractical to subject all suspected cases of IMDs to a complete set of tests. It is thus important for primary care physicians to recognize the clinical signs of and to know when to pursue advanced laboratory testing or referral to a children's subspecialty center. $8-10$

This study was conducted to assess a novel clinicobiochemical scoring system, to screen the high risk IMD cases, who were potential candidates to be subjected to further advanced laboratory testing. The present study is a preliminary report on the evaluation of this score.

\section{METHODOLOGY}

This diagnostic accuracy (validation) study was conducted in Armed Forces Institute of Pathology (AFIP), Rawalpindi, from August 2016 to August 2017. After the approval from Institutional Review Board of AFIP, Rawalpindi, 354 cases in the age group of $<1$ year to 12 years were included in the study. Patients with 
sepsis or suspected hypoxic ischemic encephalopathy were not included. Required data was collected with the help of a detailed questionnaire after taking informed consent from patients' parents. Preliminary biochemical testing including plasma ammonia, lactate, arterial blood gases (ABGs), urine for non-glucose reducing substances, and LFTs were performed in all suspected cases. Blood samples were collected in lithium heparin tubes for ammonia, and sodium fluoride tubes for plasma lactate and plasma glucose levels, and gel tubes for LFTs. Urine was collected in plain containers for ketone bodies, non-glucose reducing substances, and qualitative metabolic screening. Samples for blood gases analysis were collected in heparinised syringes. Amino acid analysis was performed on Biochrome $30+($ ) a dedicated amino acid analyser, based on ion exchange chromatography technique. Qualitative analysis of urine organic acids was done in cases strongly suspected of organic acidemia. Samples were analysed on gas chromatography, and mass spectrometry. Urine methylmalonic acid was performed only in the cases strongly suspected for organic academia on LCMS/MS, ${ }^{11}$ 6460. Results were reported in $\mathrm{mmol} / \mathrm{mol}$ of creatinine.

RISc was designed, based on 5 clinical and 5 biochemical parameters (Table I), which have high association with

Table I: RISc score card variables.

\begin{tabular}{l|c}
\hline Parameter & Score \\
\hline Consanguinity & 2 \\
Affected sibling (with similar presentation) & 2 \\
Failure to thrive & 1 \\
Jaundice & 0.5 \\
Convulsions & 1 \\
Raised plasma ammonia (>150 umol/L) & 1 \\
Urine for non-glucose reducing substances present & 1 \\
Altered acid base status & 0.5 \\
Raised plasma lactate & 0.5 \\
Deranged LFTs. & 0.5 \\
\hline Total score & 10 \\
\hline
\end{tabular}

IMDs. Each variable in the score has been assigned value according to its frequency of presentation. This card was used to triage the potential cases that will actually require advanced testing.

All the data were analysed using SPSS version 24.0. Descriptive analysis was carried out after confirming the Gaussian nature of the data by Kolmogorov-Smirnov's test of normality. Mean score and frequencies were calculated. Chi-square test and independent sample ' $t$ ' test were applied for inferential statistics where indicated. MEDCALC $\AA$ was used for calculation of various parameters of diagnostic accuracy (Table II).

\section{RESULTS}

A total of 354 cases were referred to Pediatric Metabolic Department, Armed Forces Institute of Pathology, Rawalpindi, for work up of IMDs from August 2016 to August 2017. Male to female ratio was $3: 2$, comprising of 216 males and 138 females. Median age of the patients was 1.8 years. Individual symptoms varied according to the age group, with seizures being the commonest seen in $41.5 \%$ in all the age groups, followed by developmental delay in $31.9 \%$, failure to thrive in $26.8 \%$, jaundice in $16.7 \%$, while patients had history of an affected sibling in the family. Common biochemical derangements included raised ammonia levels in $57.5 \%$, disturbed LFTs in $24.3 \%$, raised lactate levels in $21.8 \%$, altered ABGs status in $20.1 \%$, and presence of non-glucose reducing substances in $3.4 \%$. The consanguinity rate amongst parents was $67.5 \%$.

Patients were divided into three groups, based on RISc scores as low RISc (0.5-2.5), medium RISc (3.0-5.5) and high RISc (6-10). Out of the 354 cases, a total of 61 $(17.2 \%)$ patients had a RISc score of $0.5-2.5 ; 242$ $(68.4 \%)$ had RISc of 3.0-5.5; and 51 (14.4\%) had RISc of $6-10$. Thirty-three $(9.3 \%)$ patients were diagnosed with any one IMD, based on clinical history, preliminary biochemical testing and advanced testing like amino

Table II: Diagnostic accuracy of RISc score card for diagnosis of IMD.

\begin{tabular}{|c|c|c|c|c|c|c|c|c|c|}
\hline \multirow[t]{2}{*}{ RISc score card } & \multicolumn{2}{|c|}{ Diagnosis } & \multirow{2}{*}{$\begin{array}{l}\text { Sensitivity } \\
(95 \% \mathrm{Cl})\end{array}$} & \multirow{2}{*}{$\begin{array}{l}\text { Specificity } \\
(95 \% \mathrm{Cl})\end{array}$} & \multirow{2}{*}{$\begin{array}{c}\text { PLR } \\
(95 \% \mathrm{Cl})\end{array}$} & \multirow{2}{*}{$\begin{array}{c}\text { NLR } \\
(95 \% \mathrm{Cl})\end{array}$} & \multirow{2}{*}{$\begin{array}{c}\text { PPV } \\
(95 \% \mathrm{Cl})\end{array}$} & \multirow{2}{*}{$\begin{array}{c}\text { NPV } \\
(95 \% \mathrm{Cl})\end{array}$} & \multirow{2}{*}{$\begin{array}{l}\text { Accuracy } \\
(95 \% \mathrm{Cl})\end{array}$} \\
\hline & IMD +VE & IMD -ve & & & & & & & \\
\hline$\geq 6$ & $\begin{array}{c}28 \\
\text { (True positive) } \\
\end{array}$ & $\begin{array}{c}23 \\
\text { (False positive) } \\
\end{array}$ & $85 \%$ & $93 \%$ & 11.8 & 0.16 & $55 \%$ & $98 \%$ & $90 \%$ \\
\hline$<6$ & $\begin{array}{c}5 \\
\text { (False negative) }\end{array}$ & $\begin{array}{c}298 \\
\text { (Tue negative) }\end{array}$ & & & & & & & \\
\hline Total & 33 & 321 & & & & & & & \\
\hline
\end{tabular}

PLR = Positive likelihood ratio; NLR = Negative likelihood ratio; PPV = Positive predictive values; NPV = Negative predictive values.

Table III: RISc score analysis.

\begin{tabular}{|c|c|c|c|c|c|c|}
\hline \multirow[t]{2}{*}{ Diagnosis } & \multicolumn{3}{|c|}{ RISc score categories } & \multirow[t]{2}{*}{ Total } & \multirow[t]{2}{*}{ Chi-square value } & \multirow[t]{2}{*}{$p$-value } \\
\hline & $\begin{array}{c}0.5-2.5 \\
\mathrm{n}(\%)\end{array}$ & $\begin{array}{l}3-5.5 \\
n(\%)\end{array}$ & $\begin{array}{c}6-10 \\
\mathrm{n}(\%)\end{array}$ & & & \\
\hline IMD Positive & $1(3.0)$ & $4(12.1)$ & $28(84.8)$ & 33 & $103.08^{*}$ & $\mathrm{P}=0.00$ \\
\hline IMD Negative & $60(18.6)$ & $238(74.1)$ & $23(7.2)$ & 321 & & \\
\hline Total & 61 & 242 & 51 & 354 & & \\
\hline
\end{tabular}

Note: Values in parenthesis are percentages. ${ }^{*}=p$-value $<0.01$. 
acid analysis and organic acid analysis (where indicated). These included 1 (3.0\%) patient from low RISc, 4 (12.1\%) from medium RISc and 28 (84.8\%) from high RISc group, who tested positive for any one IMD. High risk group had a statistically significant higher IMD rate than the other two groups $(p<0.001$, Table III). As indicated in Table II, the sensitivity and specificity were found quite optimum, while overall accuracy of the score card was found to be $90 \%$.Comparison of mean RISc scores of IMD positive and IMD negative cases illustrated by mean plot (Figure 2).

Specific IMDs, which were diagnosed among the 354 patients, were non-ketotic hyperglycinemia in $21.2 \%$

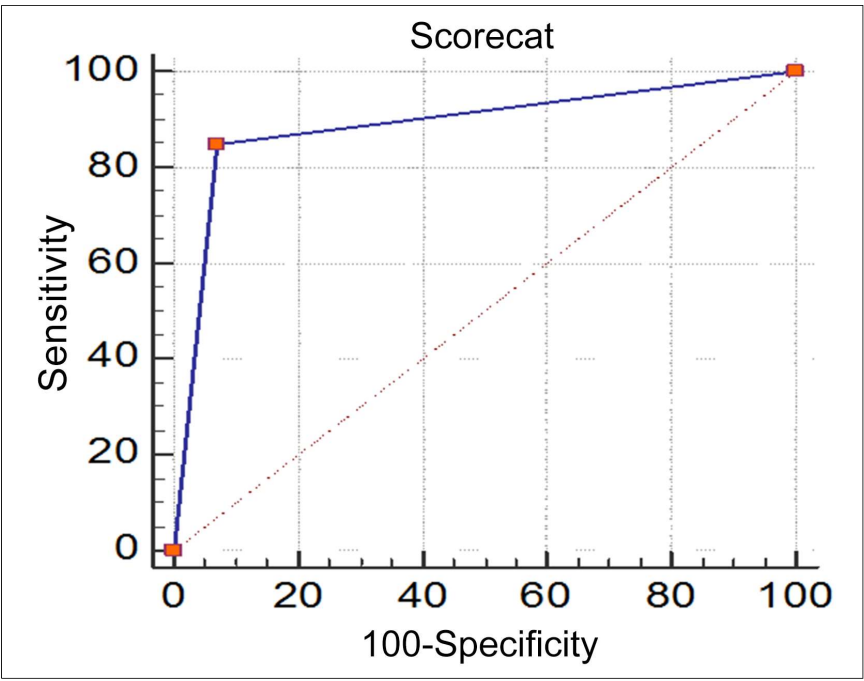

Figure 1: Roc curve at cutoff of 6 (RISc). $(n=7)$, tyrosinemia type-I in $12.2 \% \quad(n=4)$, urea cycle defects in $9.0 \%(n=3)$, organic aciduria in $9.0 \%(n=3)$, phenylketonuria, hyperprolinemia, mitochondrial disease, methylmalonic aciduria, maple syrup urine disease, hyperprolinemia in $6.1 \%(n=2)$ each; and alkaptonuria, Fanconi's syndrome, glycogen storage disease and homocystinuria $3.0 \%(n=1)$ each. ROC curve generates at cutoff level of 6 RISc score and it gives area under curve (AUC ) $0.88,85 \%$ sensitivity and $93 \%$ specificity, which shows that RISc score 6 or more is sensitive and helpful in early screening and diagnosis of IMDs (Figure 1).

\section{DISCUSSION}

IMDs are significant cause of mortality and morbidity and if undiagnosed, would affect quality of an infant's life in a developing country like Pakistan. The diagnosis involves a battery of tests often including sophisticated tests like amino acid screening, organic acids, enzyme assays and molecular testing. Due to number of socioeconomic and ethnic reasons, these cases are neglected and remained uninvestigated. The high mortality and risk of long-term neurological disability merits prompt recognition of signs and symptoms and early diagnosis. ${ }^{12}$ This is the first study of its kind where we have suggested a novel clinico-biochemical RISc system, with a unique nomenclature; and which will go a long way in costeffective screening, early diagnosis and improvement in quality of life as well as survival of new born population. Various local studies have published their data regarding IMD prevalence and pattern in the local population, but none had so far attempted to devise a score for the cost-

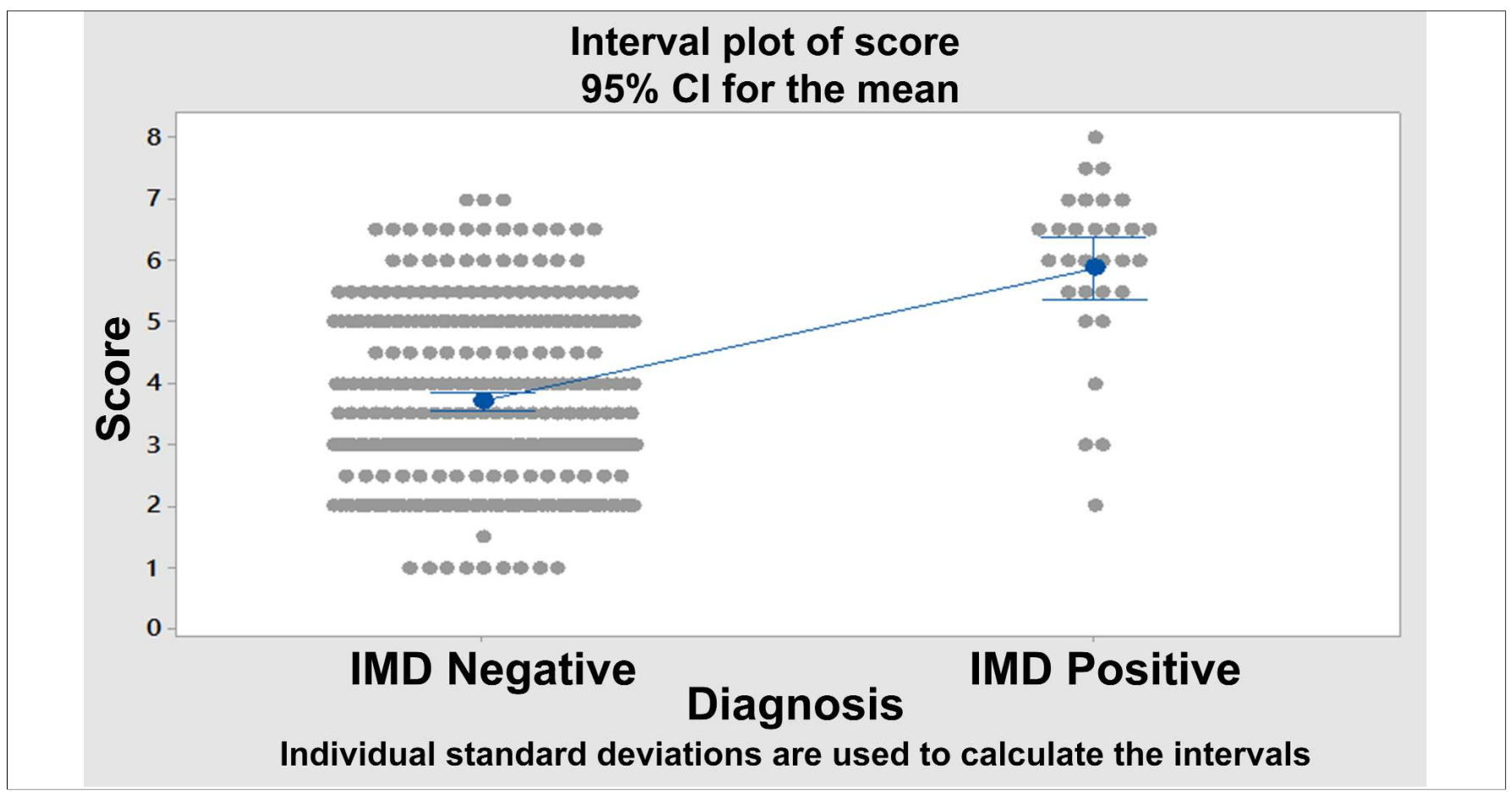

Figure 2: Comparison between mean RISc score of IMD positive and IMD negative cases. 
effective screening of suspected cases. ${ }^{13-15}$ The score system has been named as 'Rawalpindi IMD score' or RISc, corresponding to the place at which the study has been carried out; and does, by no means, imply that the sample population was restricted to any particular area of the country. In fact, we received cases from all over Pakistan.

The RISc score card suggested in the current study comprised 10 important clinical and biochemical criteria; and each was assigned a RISc number. Consanguinity constituted an important part on the RISc score card and was one of the two variables (other being affected sibling) which were assigned the highest individual score of 2. Many authors in the past have suggested that that parental consanguinity is significantly linked to autosomal recessive metabolic disorders like hyperphenylalaninemias, mucopolysaccharidosis, GM1 gangliosidosis, and glycogen storage disease type I. ${ }^{16}$ High consanguinity rate $(67.5 \%)$, much similar to seen in this study population, has also been recently reported by Sherazi et al. ${ }^{13}$

Amongst the biochemical variables, raised plasma ammonia was assigned the highest score. Levels were set at $>150 \mathrm{umol} / \mathrm{L}$. This was done to exclude other common causes of raised ammonia in infants such as sepsis. The only other cause of markedly raised ammonia levels is probably a transient hyperammonemia of newborn, but is present commonly on premature infants. ${ }^{17}$ Raised ammonia levels are in fact seen in a variety of clinical settings of IMDs like urea cycle defects, ${ }^{18}$ and organic acidemias. Presence of non-glucose reducing substances in the urine was also assigned a score of 01 (one). This very old yet very important and simple test is still being performed in many clinical laboratories worldwide, 19 and detects the presence of reducing substances like galactose, lactose, and fructose and a positive test in the setting of a suspected IMD is not only a strong evidence for the presumed condition but also warrants further testing. The test is also positive in alkaptonuria due to the presence of homogentisic acid. Another reason for assigning a higher score to this test is that in a cost restrained setting, the test can be utilised for screening so that immediate dietary intervention be made for a strongly suspected case. Amongst the 5 clinical presentations that we included on the RISc score card, presence of seizures or convulsions was assigned a relatively higher score, just as we did for patients which showed failure to thrive (Table I). Most IMDs that result in intoxication or energy deficiency are brought to attention because of rapidly progressive neurological deterioration. 20 Cheema et al. at Children Hospital, Lahore showed that $13.3 \%$ patients presented with mental retardation, ${ }^{14}$ whereas Chaudhry et al. showed that seizures and coma were the commonest presentations $(50 \%),{ }^{15}$ which is much in agreement with this data $(42 \%)$. The difference of the present data from the one reported by Cheema et al. could be attributed to the clinical setting from where patients were selected as their study was confined to only hepatology cases.

In the current study, various parameters of the score have been extensively studied by diagnostic accuracy point of view. More than $84 \%$ sensitivity and $98 \%$ NPV indicate that this score can be used for screening, while high specificity makes this a good investigation for 'rule in' of IMDs. PLR and NLR of 11.8 and 0.16 (near-zero) will help the pediatricians to assess the post-test probability of the score.

The authors suggest that cases with low RISc score be kept under observation till the symptoms settle down. Those with medium score should be followed up with plasma ammonia levels and LFTs; and those with high score, should be subjected to complex/advanced laboratory testing to reach the diagnosis. Large-scale validation of the scoring system needs to be done in the setting of diagnosis of other IMDs and this scoring system may be incorporated in a comprehensive national newborn screening programme.

Genetic markers have not been included in the study. Long term follow-up of the patients was also not carried out. A larger study with genetic markers (as reference standard) in prospective design is recommended to be carried out for detailed assessment of the score.

\section{CONCLUSION}

The cost-effective RISc card, based on clinical data and preliminary biochemical investigations is a breakthrough in diagnosing IMDs in cost restrained setups. It is strongly suggested that the initial screening for suspected IMDs and decision for advanced laboratory testing be carried out, based on the RISc card presented in the study.

\section{REFERENCES}

1. Romão A, Simon PEA, Góes JEC, Pinto LLC, Giugliani R, Luca GRd, et al. Initial clinical presentation in cases of inborn errors of metabolism in a reference children's hospital: Still a diagnostic challenge. Revista Paulista de Pediatria 2017; 35: 258-64.

2. Zhang C, Xu K, Dave UP, Wang Y, Matsumoto I. Inborn errors of metabolism discovered in Asian department of pediatrics and mental retardation research center. $J$ Chromatogr B Biomed Sci Appl 2000; 746:41-9.

3. Wolf $\mathrm{NI}$, Bast $\mathrm{T}$, Surtees R. Epilepsy in inborn errors of metabolism. Epileptic Disord 2005; 7:67-81.

4. Roy A, Samanta T, Purkait R, Mukherji A, Ganguly S. Etiology, clinical spectrum and outcome of metabolic liver diseases in children. J Coll Physicians Surg Pak 2013; 23:194-8.

5. Zwickler T, Riderer A, Haege G, Hoffmann GF, Kolker S, Burgard P. Usefulness of biochemical parameters in decisionmaking on the start of emergency treatment in patients with propionic acidemia. J Inherit Metab Dis 2014; 37:31-7. 
6. Saudubray J, de Baulny HO, Charpentier C. Clinical approach to inherited metabolic diseases. Inborn metabolic diseases: Springer; 2000. p. 3-41.

7. Hussain $R$, Bittles $A$. The prevalence and demographic characteristics of consanguineous marriages in Pakistan. J Biosoc Sci 1998; 30:261-75.

8. Karam PE, Habbal M-Z, Mikati MA, Zaatari GE, Cortas NK, Daher RT. Diagnostic challenges of aminoacidopathies and organic acidemias in a developing country: a twelve-year experience. Clin Biochem 2013; 46:1787-92.

9. Amâncio FAM, Scalco FB, Coelho CAR. Investigação diagnóstica de erros inatos do metabolismo em um hospital universitário. J Bras Patol Med Lab 2007; 43:169-74.

10. Vernon HJ. Inborn errors of metabolism: advances in diagnosis and therapy. JAMA Pediatr 2015; 169:778-82.

11. Parini $R$, Corbetta $C$. Metabolic screening for the newborn. $J$ Matern Fetal Neonatal Med 2011; 24 (Suppl 2):6-8.

12. Boyer SW, Barclay LJ, Burrage LC. Inherited metabolic disorders: Aspects of chronic nutrition management. Nutr Clin Pract 2015; 30:502-10.

13. Sherazi NA, Khan AH, Jafri L, Jamil A, Khan NA, Afroze B. Selective screening for organic acidurias and amino acidopathies in Pakistani children. J Coll Physicians Surg Pak 2017; 27:218-21.
14. Cheema HA, Malik HS, Parkash A, Fayyaz Z. Spectrum of inherited metabolic disorders in Pakistani children presenting at a tertiary care centre. J Coll Physicians Surg Pak 2016; 26: 498-502.

15. Choudhry S, Khan M, Rao HA, Jalan A, Khan EA. Etiology and outcome of inborn errors of metabolism. J Pak Med Assoc 2013; 63:1112-6.

16. Kozuki CG, Steiner CE. Consanguinity and geographic origin of patients with autosomal recessive metabolic disorders evaluated in a reference service in Campinas, Brazil. J Inborn Errors Metab Screen 2015; 3:1-5.

17. Hwang MW, Yu ST, Oh YK. A case of severe transient hyperammonemia in a newborn. Korean J Pediatr 2010; 53: 598-602.

18. Machado MC, da Silva FP. Hyperammonemia due to urea cycle disorders: a potentially fatal condition in the intensive care setting. J Intens Care 2014; 2:22.

19. Naumova NN, Schappert J, Kaplan LA. Reducing substances in urine: a paradigm for changes in a standard test. Ann Clin Lab Sci 2006; 36:447-8.

20. Sirrs S, Lehman A, Stockler S, van Karnebeek C. Treatable inborn errors of metabolism causing neurological symptoms in adults. Mol Genet Metab 2013; 110:431-8. 